\title{
Predictive Cancer Model
}

National Cancer Institute

\section{Source}

National Cancer Institute. Predictive Cancer Model. NCI Thesaurus. Code C19690.

Computer models of cancer risk designed to assess cancer risk in individuals and identifiy those at high risk, facilitate the design and planning of clinical cancer trials, foster the development of benefit-risk indices, and enable estimates of the population burden and cost of cancer. Models may also aid in the evaluation of treatments and interventions. 